\title{
NORMALITY OF FUZZY TOPOLOGICAL SPACES IN KUBIAK-ŠOSTAK'S SENSE
}

\author{
M. Azab AbD-Allah
}

\begin{abstract}
The aim of this paper is to study the normality of fuzzy topological spaces in Kubiak-Sostak's sense. Also, some characterizations and the effects of some types of functions on these types of normality are studied.
\end{abstract}

\section{Introduction}

The study of fuzzy sets was initiated with the famous paper of Zadeh [8], and thereafter Chang [1] paved the way for the subsequent tremendous growth of the numerous fuzzy topological concepts. In Chang's definition, a fuzzy topology is a crisp subfamily of family of fuzzy subsets, and fuzziness in the openness of a fuzzy subset has not been considered. An essentially more general notion of fuzzy topology, in which each fuzzy subset has a certain degree of openness, was introduced by Kubiak [5] and Šostak [8].

In [4], Krsteska and Kim defined the concepts of fuzzy generalized $\alpha$-closed sets and fuzzy generalized regular $\alpha$-closed sets in Chang's fuzzy topological space. By using the above mentioned classes of generalized fuzzy closed sets, they introduced and studied the concepts of fuzzy normal space, fuzzy almost normal space, and fuzzy mildly normal space.

In this paper, we introduce the concepts of fuzzy almost normal, fuzzy normal, fuzzy mildly normal spaces in fuzzy topological spaces in Kubiak-Šostak's sense and then, we investigate some of their characteristic properties. Our results here represent a generalization of Krsteska and Kim's study. We can simply obtain their results by taking the $r$-cut to min.

\section{Preliminaries}

Throughout this paper, let $X$ be a nonempty set and $I$ is the closed unit interval $[0,1], I_{\circ}=(0,1]$ and $I_{1}=[0,1)$. The family of all fuzzy subsets on $X$ denoted by $I^{X} \cdot \underline{0}$ and $\underline{1}$ denote the smallest and the greatest fuzzy

Received May 12, 2009

2010 Mathematics Subject Classification. 54A40.

Key words and phrases. fuzzy topology, normal fuzzy topology, almost normal, mildly normal. 
subsets on $X$, respectively. For a fuzzy subset $\lambda \in I^{X}, \underline{1}-\lambda$ denotes its complement. Given a function $f: X \rightarrow Y, f(\lambda)$ and $f^{-1}(\nu)$ define the direct image and the inverse image of $f$, defined by $f(\lambda)(y)=\bigvee_{f(x)=y} \lambda(x)$ and $f^{-1}(\nu)(x)=\nu(f(x)), \forall \nu \in I^{Y}, x \in X$, respectively. For fuzzy subsets $\lambda$ and $\mu$ in $X$, we write $\lambda q \mu$ to mean that $\lambda$ is quasi-coincident (q-coincident, for short) with $\mu$, i.e., there exists at least one point $x \in X$ such that $\lambda(x)+\mu(x)>1$. Negation of such a statement is denoted as $\lambda \bar{q} \mu$. Notions and notations not described in this paper are standard and usual.

Definition ([5, 7]). A function $\tau: I^{X} \rightarrow I$ is called a fuzzy topology on $X$ if it satisfies the following conditions:

(O1) $\tau(\underline{0})=\tau(\underline{1})=1$.

(O2) $\tau\left(\lambda_{1} \wedge \lambda_{2}\right) \geq \tau\left(\lambda_{1}\right) \wedge \tau\left(\lambda_{2}\right)$ for each $\lambda_{1}, \lambda_{2} \in I^{X}$.

(O3) $\tau\left(\vee_{i \in \Gamma} \lambda_{i}\right) \geq \wedge_{i \in \Gamma} \tau\left(\lambda_{i}\right)$ for any $\left\{\lambda_{i}\right\}_{i \in \Gamma} \subset I^{X}$.

The pair $(X, \tau)$ is called a fuzzy topological spaces (fts, for short). $\tau(\lambda)$ may be interpreted as a gradation of openness for $\lambda$. A function $f:\left(X, \tau_{1}\right) \rightarrow\left(Y, \tau_{2}\right)$ is said to be a fuzzy continuous if $\tau_{1}\left(f^{-1}(\nu)\right) \geq \tau_{2}(\nu)$ for each $\nu \in I^{Y}$.

Theorem $2.1([2])$. Let $(X, \tau)$ be an fts. Then for each $r \in I_{0}, \lambda \in I^{X}$, one defines an operator $C_{\tau}: I^{X} \times I_{0} \rightarrow I^{X}$ as follows:

$$
C_{\tau}(\lambda, r)=\bigwedge\left\{\mu \in I^{X} \mid \lambda \leq \mu, \tau\left(\mu^{\prime}\right) \geq r\right\} .
$$

For $\lambda, \mu \in I^{X}$ and $r, s \in I_{0}$, the operator $C_{\tau}$ satisfies the following statements:

(C1) $C_{\tau}(\underline{0}, r)=\underline{0}$.

(C2) $\lambda \leq C_{\tau}(\lambda, r)$.

(C3) $C_{\tau}(\lambda, r) \vee C_{\tau}(\mu, r)=C_{\tau}(\lambda \vee \mu, r)$.

(C4) $C_{\tau}(\lambda, r) \leq C_{\tau}(\lambda, s)$ if $r \leq s$.

(C5) $C_{\tau}\left(C_{\tau}(\lambda, r), r\right)=C_{\tau}(\lambda, r)$.

Theorem $2.2([3])$. Let $(X, \tau)$ be an fts. Then for each $r \in I_{0}$ and $\lambda \in I^{X}$, one defines an operator $I_{\tau}: I^{X} \times I_{1} \rightarrow I^{X}$ as follows:

$$
I_{\tau}(\lambda, r)=\bigvee\left\{\mu \in I^{X} \mid \mu \leq \lambda, \tau(\mu) \geq r\right\} .
$$

For $\lambda, \mu \in I^{X}$ and $r, s \in I_{0}$, the operator $I_{\tau}$ satisfies the following statements:

(I1) $I_{\tau}(\underline{1}-\lambda, r)=\underline{1}-C_{\tau}(\lambda, r)$.

(I2) $I_{\tau}(\underline{1}, r)=\underline{1}$.

(I3) $I_{\tau}(\lambda, r) \leq \lambda$.

(I4) $I_{\tau}(\lambda, r) \wedge I_{\tau}(\mu, r)=I_{\tau}(\lambda \wedge \mu, r)$.

(I5) $I_{\tau}(\lambda, s) \leq I_{\tau}(\lambda, r)$ if $r \leq s$.

(I6) $I_{\tau}\left(I_{\tau}(\lambda, r), r\right)=I_{\tau}(\lambda, r)$.

(I7) If $I_{\tau}\left(C_{\tau}(\lambda, r), r\right)=\lambda$, then $C_{\tau}\left(I_{\tau}(\underline{1}-\lambda, r), r\right)=\underline{1}-\lambda$.

Definition ([6]). Let $(X, \tau)$ be an fts, $\lambda \in I^{X}$ and $r \in I_{0}$.

(1) A fuzzy set $\lambda$ is called $r$-regular fuzzy open (for short, $r$-rfo) if $\lambda=$ $I_{\tau}\left(C_{\tau}(\lambda, r), r\right)$. 
(2) A fuzzy set $\lambda$ is called $r$-regular fuzzy closed (for short, $r$-rfc) if $\lambda=$ $C_{\tau}\left(I_{\tau}(\lambda, r), r\right)$.

(3) A fuzzy set $\lambda$ is called $r$-fuzzy $\alpha$-open (for short, $r$-f $\alpha$ o) if

$$
\lambda \leq I_{\tau}\left(C_{\tau}\left(I_{\tau}(\lambda, r), r\right), r\right) .
$$

$\lambda$ is called $r$-fuzzy $\alpha$-closed (for short, $r$-f $\alpha \mathrm{c}$ ) if and only if $\underline{1}-\lambda$ is $r$-f $\alpha$ o. The $r$-fuzzy $\alpha$-closure and $r$-fuzzy $\alpha$-interior of $\lambda$ is defined by

$$
\begin{aligned}
& \alpha C_{\tau}(\lambda, r)=\bigwedge\left\{\mu \in I^{X} \mid \lambda \leq \mu, \mu \text { is } r \text { - } \alpha \mathrm{fc}\right\}, \\
& \alpha I_{\tau}(\lambda, r)=\bigvee\left\{\mu \in I^{X} \mid \mu \leq \lambda, \mu \text { is } r \text { - } \alpha \mathrm{fo}\right\} .
\end{aligned}
$$

Definition. Let $(X, \tau)$ be an fts, $\lambda, \mu \in I^{X}$ and $r \in I_{0}$.

(1) A fuzzy set $\lambda$ is called $r$-generalized fuzzy closed (for short, $r$-gfc) if $C_{\tau}(\lambda, r) \leq \mu$ whenever $\lambda \leq \mu$ and $\tau(\mu) \geq r . \lambda$ is called $r$-generalized fuzzy open (for short, $r$-gfo) if $\underline{1}-\lambda$ is $r$-generalized fuzzy closed.

(2) A fuzzy set $\lambda$ is called $r$-generalized regular fuzzy closed (for short, $r$-grfc) if $C_{\tau}(\lambda, r) \leq \mu$ whenever $\lambda \leq \mu$ and $\mu$ is $r$-rfo. $\lambda$ is called $r$ generalized regular fuzzy open (for short, $r$-grfo) if and only if $\underline{1}-\lambda$ is $r$-grfc.

(3) A fuzzy set $\lambda$ is called $r$-generalized fuzzy $\alpha$-closed (for short, $r$-gf $\alpha \mathrm{c}$ ) if $\alpha C_{\tau}(\lambda, r) \leq \mu$ whenever $\lambda \leq \mu$ and $\tau(\mu) \geq r . \lambda$ is called $r$-generalized fuzzy $\alpha$-open (for short, $r$-gf $\alpha \mathrm{O}$ ) if and only if $\underline{1}-\lambda$ is $r$-gf $\alpha \mathrm{c}$.

(4) A fuzzy set $\lambda$ is called $r$-generalized regular fuzzy $\alpha$-closed (for short, $r$-grf $\alpha \mathrm{c})$ if $\alpha C_{\tau}(\lambda, r) \leq \mu$ whenever $\lambda \leq \mu$ and $\mu$ is $r$-rfo. $\lambda$ is called $r$-generalized regular fuzzy $\alpha$-open (for short, $r$-grf $\alpha \mathrm{o}$ ) if and only if $1-\lambda$ is $r$-grf $\alpha \mathrm{c}$.

Remark 2.3. From the above definitions it is not difficult to conclude that the following diagram of implications is true:

$$
\begin{array}{ccc}
r \text {-gfc set } & \Rightarrow & r \text {-grfc set } \\
\Downarrow & & \Downarrow \\
r \text {-gf } \alpha \text { c set } & \Rightarrow & r \text {-grf } \alpha \text { c set }
\end{array}
$$

Counter example 2.1. Let $X=\{a, b\}$ and let $\lambda_{1}, \mu_{1}$ and $\nu_{1}$ are fuzzy sets defined by

$$
\begin{array}{ll}
\lambda_{1}(a)=0.2, & \lambda_{1}(b)=0.4 ; \\
\mu_{1}(a)=0.9, & \mu_{1}(b)=0.4 ; \\
\nu_{1}(a)=0.1, & \nu_{1}(b)=0.4 .
\end{array}
$$

Define $\tau_{1}$ and $\tau_{2}$ on $X$ as follows:

$$
\tau_{1}(\lambda)=\left\{\begin{array}{ll}
1, & \text { if } \lambda=\underline{0}, \underline{1} ; \\
\frac{1}{4}, & \text { if } \lambda=\lambda_{1} ; \\
\frac{1}{3}, & \text { if } \lambda=\mu_{1} ; \\
0, & \text { otherwise, }
\end{array} \quad \tau_{2}(\lambda)= \begin{cases}1, & \text { if } \lambda=\underline{0}, \underline{1} ; \\
\frac{1}{2}, & \text { if } \lambda=\mu_{1} ; \\
0, & \text { otherwise }\end{cases}\right.
$$


(1) In $\tau_{1}$, it can be shown that $\nu_{1}$ is an $\frac{1}{4}$-gf $\alpha \mathrm{c}$ set, but it is not an $\frac{1}{4}$-gfc set. Also, $\nu_{1}$ is an $\frac{1}{4}$-grf $\alpha$ c set, but it is not an $\frac{1}{4}$-grfc set.

(2) In $\tau_{2}, \nu_{1}$ is an $\frac{1}{2}$-grf $\alpha \mathrm{c}$ set, but it is not an $\frac{1}{2}$-gf $\alpha \mathrm{c}$ set. Also, $\nu_{1}$ is an $\frac{1}{2}$-grfc set, but it is not an $\frac{1}{2}$-gfc set.

Definition. Let $f:\left(X, \tau_{1}\right) \rightarrow\left(Y, \tau_{2}\right)$ be a function between $\mathrm{fts}$ 's $\left(X, \tau_{1}\right)$ and $\left(Y, \tau_{2}\right)$. Then the function $f$ is called:

(1) fuzzy regular continuous if $f^{-1}(\nu)$ is $r$-rfo for each $\nu \in I^{Y}$ and $r \in I_{0}$ such that $\tau_{2}(\nu) \geq r$.

(2) fuzzy regular irresolute if $f^{-1}(\nu)$ is $r$-rfo for each $\nu \in I^{Y}, r \in I_{0}$ such that $\nu$ is $r$-rfo.

(3) fuzzy almost regular generalized continuous if $f^{-1}(\nu)$ is $r$-grfo for each $\nu \in I^{Y}$ and $r \in I_{0}$ such that $\tau_{2}(\nu) \geq r$.

(4) fuzzy almost continuous if $\tau_{1}\left(f^{-1}(\nu)\right) \geq r$ for each $\nu \in I^{Y}, r \in I_{0}$ such that $\nu$ is $r$-rfo.

Definition. Let $f:\left(X, \tau_{1}\right) \rightarrow\left(Y, \tau_{2}\right)$ be a function between $\mathrm{fts}$ 's $\left(X, \tau_{1}\right)$ and $\left(Y, \tau_{2}\right)$. Then the function $f$ is called:

(1) fuzzy regular closed if $f(\lambda)$ is $r$-rfc for each $\lambda \in I^{X}, r \in I_{0}$ such that $\tau_{1}\left(\lambda^{\prime}\right) \geq r$.

(2) fuzzy almost closed if $\tau_{2}\left(f(\lambda)^{\prime}\right) \geq r$ for each $\lambda \in I^{X}, r \in I_{0}$ such that $\lambda$ is $r$-rfc.

(3) fuzzy almost generalized closed if $f(\lambda)$ is $r$-gfc for each $\lambda \in I^{X}, r \in I_{0}$ such that $\lambda$ is $r$-rfc.

(4) fuzzy almost regular generalized closed if $f(\lambda)$ is $r$-grfc for each $\lambda \in I^{X}$, $r \in I_{0}$ such that $\lambda$ is $r$-rfc.

\section{Fuzzy normal spaces}

Definition. An fts $(X, \tau)$ is said to be:

(1) fuzzy almost normal space if for each $\lambda_{1}, \lambda_{2} \in I^{X}$ and $r \in I_{0}$ such that $\tau\left(\lambda_{1}^{\prime}\right) \geq r, \lambda_{2}$ is $r$-rfc set and $\lambda_{1} \bar{q} \lambda_{2}$, there exist $\mu_{1}, \mu_{2} \in I^{X}$ such that $\lambda_{1} \leq \mu_{1}, \lambda_{2} \leq \mu_{2}$ with $\mu_{1} \bar{q} \mu_{2}$.

(2) fuzzy normal space if for each $\lambda_{1}, \lambda_{2} \in I^{X}$ and $r \in I_{0}$ such that $\tau\left(\lambda_{1}^{\prime}\right) \geq$ $r, \tau\left(\lambda_{2}^{\prime}\right) \geq r$ and $\lambda_{1} \bar{q} \lambda_{2}$, there exists $\mu_{1}, \mu_{2} \in I^{X}$ such that $\tau\left(\mu_{1}\right) \geq r$, $\tau\left(\mu_{2}\right) \geq r, \lambda_{1} \leq \mu_{1}, \lambda_{2} \leq \mu_{2}$ and $\mu_{1} \bar{q} \mu_{2}$.

(3) fuzzy mildly normal space if for each $\lambda_{1}, \lambda_{2} \in I^{X}$ and $r \in I_{0}$ such that $\lambda_{1}$ and $\lambda_{2}$ are $r$-rfc sets and $\lambda_{1} \bar{q} \lambda_{2}$, there exists $\mu_{1}, \mu_{2} \in I^{X}$ such that $\tau\left(\mu_{1}\right) \geq r, \tau\left(\mu_{2}\right) \geq r, \lambda_{1} \leq \mu_{1}, \lambda_{2} \leq \mu_{2}$ and $\mu_{1} \bar{q} \mu_{2}$.

Clearly, every fuzzy normal space is almost fuzzy normal space and every fuzzy almost normal space is fuzzy mildly normal space, but the converse is not true in general as the following example shows: 
Example 3.1. Let $X=\{x\}$ and define the fuzzy sets $\mu, \nu, \rho, \gamma$ and $\omega$ as follows:

$$
\mu(x)=0.4, \quad \nu(x)=0.7, \quad \rho(x)=0.8, \quad \gamma(x)=0.6, \quad \omega(x)=0.2 .
$$

Define the fuzzy topologies $\tau_{1}, \tau_{2}$ as follows:

$$
\begin{gathered}
\tau_{1}(\lambda)= \begin{cases}1, & \text { if } \lambda=\underline{0}, \underline{1} ; \\
\frac{1}{4}, & \text { if } \lambda=\mu ; \\
\frac{1}{3}, & \text { if } \lambda=\nu ; \\
0, & \text { otherwise. }\end{cases} \\
\tau_{2}(\lambda)= \begin{cases}1, & \text { if } \lambda=\underline{0}, \underline{1} ; \\
\frac{1}{4}, & \text { if } \lambda=\nu ; \\
\frac{1}{2}, & \text { if } \lambda=\rho ; \\
\frac{1}{5}, & \text { if } \lambda=\gamma ; \\
\frac{1}{6}, & \text { if } \lambda=\omega ; \\
0, & \text { otherwise. }\end{cases}
\end{gathered}
$$

(1) $\left(X, \tau_{1}\right)$ is fuzzy mildly normal space but not fuzzy almost normal space.

(2) $\left(X, \tau_{2}\right)$ is fuzzy almost normal space but not fuzzy normal space.

Theorem 3.1. Let $(X, \tau)$ be an fts. Then the following statements are equivalent:

(1) $(X, \tau)$ is a fuzzy mildly normal space;

(2) For each pair of $r$-rfc sets $\lambda_{1}, \lambda_{2} \in I^{X}$ and $r \in I_{0}$ such that $\lambda_{1} \bar{q} \lambda_{2}$, there exist $r$-rfo sets $\mu_{1}, \mu_{2}$ such that $\lambda_{1} \leq \mu_{1}, \lambda_{2} \leq \mu_{2}$ and $\mu_{1} \bar{q} \mu_{2}$;

(3) For each pair of $r$-rfc sets $\lambda_{1}, \lambda_{2} \in I^{X}$ and $r \in \bar{I}_{0}$ such that $\lambda_{1} \bar{q} \lambda_{2}$, there exist $r$-gfo sets $\mu_{1}, \mu_{2}$ such that $\lambda_{1} \leq \mu_{1}, \lambda_{2} \leq \mu_{2}$ and $\mu_{1} \bar{q} \mu_{2}$;

(4) For each pair of $r$-rfc sets $\lambda_{1}, \lambda_{2} \in I^{X}$ and $r \in I_{0}$ such that $\lambda_{1} \bar{q} \lambda_{2}$, there exist $r$-gfoo sets $\mu_{1}$ and $\mu_{2}$, such that $\lambda_{1} \leq \mu_{1}, \lambda_{2} \leq \mu_{2}$ and $\mu_{1} \bar{q} \mu_{2}$;

(5) For each $r$-rfc set $\lambda \in I^{X}$ and each $r$-rfo set $\mu \in I^{X}$ such that $\lambda \leq \mu$, there exists $\rho \in I^{X}$ such that $\tau(\rho) \geq r$ and

$$
\lambda \leq \rho \leq C_{\tau}(\rho, r) \leq \mu
$$

(6) For each $r$-rfc set $\lambda \in I^{X}$ and each $r$-rfo set $\mu \in I^{X}$ such that $\lambda \leq \mu$, there an $r$-rfo set $\rho \in I^{X}$ such that

$$
\lambda \leq \rho \leq C_{\tau}(\rho, r) \leq \mu
$$

(7) For each $r$-rfc set $\lambda \in I^{X}$ and $r$-rfo set $\mu \in I^{X}$ such that $\lambda \leq \mu$, there exists an $r$-gfo set $\rho \in I^{X}$ such that

$$
\lambda \leq \rho \leq C_{\tau}(\rho, r) \leq \mu
$$

(8) For each $r$-rfc set $\lambda \in I^{X}$ and each $r$-rfo set $\mu \in I^{X}$ such that $\lambda \leq \mu$, there exists an $r$-gfoo set $\rho \in I^{X}$ such that

$$
\lambda \leq \rho \leq \alpha C_{\tau}(\rho, r) \leq \mu
$$


(9) For each $r$-rfc set $\lambda \in I^{X}$ and each $r$-rfo set $\mu \in I^{X}$ such that $\lambda \leq \mu$, there exists an $r$-foo set $\rho \in I^{X}$ such that

$$
\lambda \leq \rho \leq \alpha C_{\tau}(\rho, r) \leq \mu
$$

(10) For each $r$-rfc sets $\lambda_{1}, \lambda_{2} \in I^{X}$ and $r \in I_{0}$ such that $\lambda_{1} \bar{q} \lambda_{2}$, there exist $r$-f $\alpha$ o sets $\mu_{1}$ and $\mu_{2}$, such that $\lambda_{1} \leq \mu_{1}, \lambda_{2} \leq \mu_{2}$ and $\mu_{1} \bar{q} \mu_{2}$.

Proof. $(1) \Rightarrow(5)$ For each $r \in I_{0}$, let $\lambda$ be $r$-rfc set and let $\mu$ be an $r$-rfo set such that $\lambda \leq \mu$. Then $\lambda \bar{q} \underline{1}-\mu$. By (1), there exist an $r$-rfo sets $\lambda_{1}$ and $\mu_{1}$, such that $\lambda \leq \lambda_{1}, \underline{1}-\mu \leq \lambda_{1}$ such that $\lambda_{1} \bar{q} \mu_{1}$. Thus

$$
\lambda \leq \lambda_{1} \leq C_{\tau}\left(\lambda_{1}, r\right) \leq \underline{1}-\mu_{1} \leq \mu .
$$

$(5) \Rightarrow(2)$ Let $\lambda_{1}$ and $\lambda_{2}$ be an $r$-rfc set such that $\lambda_{1} \bar{q} \lambda_{2}$. Then $\lambda_{1} \leq \underline{1}-\lambda_{2}$. By (5), there exists $\rho \in I^{X}$ such that $\tau(\rho) \geq r$ and

$$
\lambda_{1} \leq \rho \leq C_{\tau}(\rho, r) \leq \underline{1}-\lambda_{2} .
$$

It follows that

$$
\lambda_{1} \leq I_{\tau}\left(C_{\tau}(\rho, r), r\right) \leq \underline{1}-\lambda_{2}
$$

and

$$
\lambda_{2} \leq \underline{1}-C_{\tau}(\rho, r)=I_{\tau}(\underline{1}-\rho, r) .
$$

Then $\mu_{1}=I_{\tau}\left(C_{\tau}(\rho, r), r\right)$ and $\mu_{2}=I_{\tau}(\underline{1}-\rho, r)$ are $r$-rfo sets such that $\lambda_{1} \leq \mu_{1}$, $\lambda_{2} \leq \mu_{2}$ and $\mu_{1} \bar{q} \mu_{2}$.

$(2) \Rightarrow(6)$ Let $\lambda_{1}$ be any $r$-rfc set and let $\lambda_{2}$ be $r$-rfo set such that $\lambda_{1} \leq \lambda_{2}$. Then $\lambda_{1} \bar{q} \underline{1}-\lambda_{2}$. According to (2), there exist $r$-rfo sets $\mu_{1}$ and $\mu_{2}$ such that $\lambda_{1} \leq \mu_{1}, \underline{1}-\lambda_{2} \leq \mu_{2}$ and $\mu_{1} \bar{q} \mu_{2}$. Let $\rho=I_{\tau}\left(C_{\tau}\left(\mu_{1}, r\right), r\right)$. Then $\rho$ is an $r$-rfo set such that

$$
\lambda_{1} \leq \rho \leq C_{\tau}(\rho, r) \leq \underline{1}-\mu_{2} \leq \lambda_{2} .
$$

$(6) \Rightarrow(2)$ Let $\lambda_{1}$ and $\lambda_{2}$ be $r$-rfc sets such that $\lambda_{1} \bar{q} \lambda_{2}$. Then $\lambda_{1} \leq \underline{1}-\lambda_{2}$. By $(6)$, there exists $r$-rfo set $\rho$ such that

$$
\lambda_{1} \leq \rho \leq C_{\tau}(\rho, r) \leq \underline{1}-\lambda_{2} .
$$

For $C_{\tau}(\rho, r) \leq \underline{1}-\lambda_{2}$, there exists $r$-rfo set $\mu$ such that

$$
C_{\tau}(\rho, r) \leq \mu \leq C_{\tau}(\mu, r) \leq \underline{1}-\lambda_{2} .
$$

Then $\lambda_{2} \leq \underline{1}-C_{\tau}(\mu, r)=I_{\tau}(\underline{1}-\mu, r), I_{\tau}(\underline{1}-\mu, r)$ is $r$-rfo set and $\rho \bar{q} I_{\tau}(\underline{1}-\mu, r)$.

(4) $\Rightarrow(8)$ Let $\lambda_{1}$ be an $r$-rfc set and let $\lambda_{2}$ be $r$-rfo set such that $\lambda_{1} \leq \lambda_{2}$. Then $\lambda_{1} \bar{q} \underline{1}-\lambda_{2}$. By (4), there exist $r$-gf $\alpha$ o sets $\mu_{1}$ and $\mu_{2}$ such that $\lambda_{1} \leq \mu_{1}$, $\underline{1}-\lambda_{2} \leq \mu_{2}$ and $\mu_{1} \bar{q} \mu_{2}$. Since $\mu_{2}$ is $r$-gf $\alpha \mathrm{o}$ set and $\underline{1}-\lambda_{2}$ is $r$-rfc set, from $\underline{1}-\lambda_{2} \leq \mu_{2}$ follows that $\underline{1}-\lambda_{2} \leq \alpha I_{\tau}\left(\mu_{2}, r\right)$. Thus

$$
\underline{1}-\lambda_{2} \leq \alpha I_{\tau}\left(\mu_{2}, r\right) \leq \mu_{2} \leq \underline{1}-\mu_{1} .
$$

Since $\underline{1}-I_{\tau}\left(\mu_{2}\right)$ is an $r$-gf $\alpha$ c set, from $\mu_{1} \leq \underline{1}-\alpha I_{\tau}\left(\mu_{2}, r\right)$, we obtain that

$$
\alpha C_{\tau}\left(\mu_{1}, r\right) \leq \underline{1}-\alpha I_{\tau}\left(\mu_{2}, r\right) .
$$

Hence

$$
\lambda_{1} \leq \mu_{1} \leq \alpha C_{\tau}\left(\mu_{1}, r\right) \leq \lambda_{2} .
$$


$(8) \Rightarrow(9)$ Let $\lambda_{1}$ be an $r$-rfc set and let $\lambda_{2}$ be an $r$-rfo set such that $\lambda_{1} \leq \lambda_{2}$. Then $\lambda_{1} \bar{q} \underline{1}-\mu$. By (8), there exists an $r$-gf $\alpha$ o set $\mu_{1}$ such that

$$
\lambda_{1} \leq \mu_{1} \leq \alpha C_{\tau}\left(\mu_{1}, r\right) \leq \lambda_{2} .
$$

Since $\mu_{1}$ is an $r$-gf $\alpha$ o set, from $\lambda_{1} \leq \mu_{1}$ follows that $\lambda_{1} \leq \alpha I_{\tau}\left(\mu_{1}, r\right)$. Then, $\mu_{2}=\alpha I_{\tau}\left(\mu_{1}, r\right)$ is an $r$-f $\alpha$ o set and

$$
\lambda_{1} \leq \mu_{2} \leq \alpha C_{\tau}\left(\mu_{2}, r\right) \leq \alpha C_{\tau}\left(\mu_{1}, r\right) \leq \lambda_{2} .
$$

$(9) \Rightarrow(10)$ Let $\lambda_{1}$ and $\lambda_{2}$ be are $r$-rfc sets such that $\lambda_{1} \bar{q} \lambda_{2}$. Then $\lambda_{1} \leq \underline{1}-\lambda_{2}$. By (9), there exists $r$-f $\alpha$ o set $\mu_{1}$ such that

$$
\lambda_{1} \leq \mu_{1} \leq \alpha C_{\tau}\left(\mu_{1}, r\right) \leq \underline{1}-\lambda_{2} .
$$

Then $\mu_{2}=\underline{1}-\alpha C_{\tau}\left(\mu_{1}, r\right)$ is an $r$-f $\alpha$ o set and $\mu_{1} \bar{q} \mu_{2}$.

$(10) \Rightarrow(1)$ Let $\lambda_{1}$ and $\lambda_{2}$ be are $r$-rfc sets such that $\lambda_{1} \bar{q} \lambda_{2}$. Then $\lambda_{1} \leq \underline{1}-\lambda_{2}$. By (10), there exist $r$-f $\alpha$ o sets $\mu_{1}$ and $\mu_{2}$ such that $\lambda_{1} \leq \mu_{1}, \lambda_{2} \leq \mu_{2}$ and $\mu_{1} \bar{q} \mu_{2}$. Let $\rho_{1}=I_{\tau}\left(C_{\tau}\left(I_{\tau}\left(\mu_{1}, r\right), r\right), r\right)$ and $\rho_{2}=I_{\tau}\left(C_{\tau}\left(I_{\tau}\left(\mu_{2}, r\right), r\right), r\right)$. Then $\tau\left(\rho_{1}\right) \geq r$ and $\tau\left(\rho_{2}\right) \geq r$ and $\rho_{1} \bar{q} \rho_{2}$. Hence $(X, \tau)$ is a fuzzy normal space.

The implications $(1) \Rightarrow(3) \Rightarrow(4),(3) \Rightarrow(7) \Rightarrow(8)$ and $(2) \Rightarrow(1)$ are trivial.

Theorem 3.2. Let $(X, \tau)$ be an fts. Then the following statements are equivalent:

(1) $(X, \tau)$ is a fuzzy normal space (resp. fuzzy almost normal space);

(2) For each $\lambda_{1}, \lambda_{2} \in I^{X}$ and $r \in I_{0}$ such that $\tau\left(\lambda_{1}^{\prime}\right) \geq r, \tau\left(\lambda_{2}^{\prime}\right) \geq r$ and $\lambda_{1} \bar{q} \lambda_{2}$, there exist $r$-rfo sets $\mu_{1}, \mu_{2}$ such that $\lambda_{1} \leq \mu_{1}, \lambda_{2} \leq \mu_{2}$ and $\mu_{1} \bar{q} \mu_{2}$;

(3) For each $\lambda_{1}, \lambda_{2} \in I^{X}$ and $r \in I_{0}$ such that $\tau\left(\lambda_{1}^{\prime}\right) \geq r, \tau\left(\lambda_{2}^{\prime}\right) \geq r$ and $\lambda_{1} \bar{q} \lambda_{2}$, there exist $r$-gfo sets $\mu_{1}, \mu_{2}$ such that $\lambda_{1} \leq \mu_{1}, \lambda_{2} \leq \mu_{2}$ and $\mu_{1} \bar{q} \mu_{2}$;

(4) For each $\lambda_{1}, \lambda_{2} \in I^{X}$ and $r \in I_{0}$ such that $\tau\left(\lambda_{1}^{\prime}\right) \geq r, \tau\left(\lambda_{2}^{\prime}\right) \geq r$ and $\lambda_{1} \bar{q} \lambda_{2}$, there exist $r-g$ foo sets $\mu_{1}$ and $\mu_{2}$, such that $\lambda_{1} \leq \mu_{1}, \lambda_{2} \leq \mu_{2}$ and $\mu_{1} \bar{q} \mu_{2}$;

(5) For each $\lambda_{1}, \lambda_{2} \in I^{X}$ and $r \in I_{0}$ such that $\tau\left(\lambda_{1}^{\prime}\right) \geq r, \tau\left(\lambda_{2}\right) \geq r$ and $\lambda_{1} \leq \lambda_{2}$, there exists $\rho \in I^{X}$ such that $\tau(\rho) \geq r$ and

$$
\lambda_{1} \leq \rho \leq C_{\tau}(\rho, r) \leq \lambda_{2}
$$

(6) For each $\lambda_{1}, \lambda_{2} \in I^{X}$ and $r \in I_{0}$ such that $\tau\left(\lambda_{1}^{\prime}\right) \geq r, \tau\left(\lambda_{2}\right) \geq r$ and $\lambda_{1} \leq \lambda_{2}$, there an $r$-rfo set $\rho \in I^{X}$ such that

$$
\lambda_{1} \leq \rho \leq C_{\tau}(\rho, r) \leq \lambda_{2} ;
$$

(7) For each $\lambda_{1}, \lambda_{2} \in I^{X}$ and $r \in I_{0}$ such that $\tau\left(\lambda_{1}^{\prime}\right) \geq r, \tau\left(\lambda_{2}\right) \geq r$ and $\lambda_{1} \leq \lambda_{2}$, there exists an $r$-gfo set $\rho \in I^{X}$ such that

$$
\lambda_{1} \leq \rho \leq C_{\tau}(\rho, r) \leq \lambda_{2}
$$


(8) For each $\lambda_{1}, \lambda_{2} \in I^{X}$ and $r \in I_{0}$ such that $\tau\left(\lambda_{1}^{\prime}\right) \geq r, \tau\left(\lambda_{2}\right) \geq r$ and $\lambda_{1} \leq \lambda_{2}$, there exists an $r$-gfoo set $\rho \in I^{X}$ such that

$$
\lambda_{1} \leq \rho \leq \alpha C_{\tau}(\rho, r) \leq \lambda_{2}
$$

(9) For each $\lambda_{1}, \lambda_{2} \in I^{X}$ and $r \in I_{0}$ such that $\tau\left(\lambda_{1}^{\prime}\right) \geq r, \tau\left(\lambda_{2}\right) \geq r$ and $\lambda_{1} \leq \lambda_{2}$, there exists an $r$-f $\alpha$ o set $\rho \in I^{X}$ such that

$$
\lambda_{1} \leq \rho \leq \alpha C_{\tau}(\rho, r) \leq \lambda_{2} ;
$$

(10) For each $\lambda_{1}, \lambda_{2} \in I^{X}$ and $r \in I_{0}$ such that $\tau\left(\lambda_{1}^{\prime}\right) \geq r, \tau\left(\lambda_{2}^{\prime}\right) \geq r$ and $\lambda_{1} \bar{q} \lambda_{2}$, there exist $r$-f $\alpha$ o sets $\mu_{1}$ and $\mu_{2}$, such that $\lambda_{1} \leq \mu_{1}, \lambda_{2} \leq \mu_{2}$ and $\mu_{1} \bar{q} \mu_{2}$.

Proof. It is clear from Theorem 3.1.

Theorem 3.3. Let $\left(X, \tau_{1}\right)$ and $\left(Y, \tau_{2}\right)$ be fts's such that $\left(X, \tau_{1}\right)$ is a fuzzy normal space. If $f:\left(X, \tau_{1}\right) \rightarrow\left(Y, \tau_{2}\right)$ is a fuzzy almost continuous, fuzzy almost closed and surjective function, then $\left(Y, \tau_{2}\right)$ is a fuzzy mildly normal space.

Proof. For each $r \in I_{0}$, let $\nu_{1}, \nu_{2} \in I^{Y}$ be $r$-rfc sets such that $\nu_{1} \bar{q} \nu_{2}$. Since $f$ is a fuzzy almost continuous function, $\tau_{1}\left(f^{-1}\left(\nu_{1}\right)^{\prime}\right) \geq r, \tau_{1}\left(f^{-1}\left(\nu_{2}\right)^{\prime}\right) \geq r$ and $f^{-1}\left(\nu_{1}\right) \bar{q} f^{-1}\left(\nu_{2}\right)$. Since $\left(X, \tau_{1}\right)$ is a fuzzy normal space, there exist $\mu_{1}, \mu_{2} \in I^{X}$ such that $\tau_{1}\left(\mu_{1}\right) \geq r, \tau_{1}\left(\mu_{2}\right) \geq r$ and $f^{-1}\left(\nu_{1}\right) \leq \mu_{1}, f^{-1}\left(\nu_{2}\right) \leq \mu_{2}$ with $\mu_{1} \bar{q} \mu_{2}$. Since $I_{\tau_{1}}\left(C_{\tau_{1}}\left(\mu_{1}, r\right), r\right)$ and $I_{\tau_{1}}\left(C_{\tau_{1}}\left(\mu_{2}, r\right), r\right)$ are $r$-rfo sets and

$$
I_{\tau_{1}}\left(C_{\tau_{1}}\left(\mu_{1}, r\right), r\right) \bar{q} I_{\tau_{1}}\left(C_{\tau_{1}}\left(\mu_{2}, r\right), r\right) .
$$

Furthermore,

$$
f^{-1}\left(\nu_{i}\right) \leq \mu_{i} \leq I_{\tau_{1}}\left(C_{\tau_{1}}\left(\mu_{i}, r\right), r\right) \text { for each } i \in\{1,2\} .
$$

Since $f$ is fuzzy almost closed, there exist $\gamma_{1}, \gamma_{2} \in I^{Y}$ such that $\tau_{2}\left(\gamma_{1}\right) \geq r$, $\tau_{2}\left(\gamma_{2}\right) \geq r$ and $\nu_{1} \leq \gamma_{1}, \nu_{2} \leq \gamma_{2}$ with

$$
f^{-1}\left(\gamma_{i}\right) \leq I_{\tau_{1}}\left(C_{\tau_{1}}\left(\mu_{i}, r\right), r\right) \text { for each } i \in\{1,2\} .
$$

Moreover $\gamma_{1} \bar{q} \gamma_{2}$. Hence $\left(Y, \tau_{2}\right)$ is a fuzzy mildly normal space.

Corollary 3.4. Let $\left(X, \tau_{1}\right)$ and $\left(Y, \tau_{2}\right)$ be fuzzy topological spaces and let $\left(X, \tau_{1}\right)$ be a fuzzy normal space. If $f:\left(X, \tau_{1}\right) \rightarrow\left(Y, \tau_{2}\right)$ is a fuzzy almost continuous and fuzzy closed function, then $\left(Y, \tau_{2}\right)$ is a fuzzy mildly normal space.

Corollary 3.5. Let $\left(X, \tau_{1}\right)$ and $\left(Y, \tau_{2}\right)$ be fuzzy topological spaces and let $\left(X, \tau_{1}\right)$ be fuzzy mildly normal space. If $f:\left(X, \tau_{1}\right) \rightarrow\left(Y, \tau_{2}\right)$ is a fuzzy almost continuous, fuzzy almost closed and fuzzy open (resp. fuzzy continuous, fuzzy closed, fuzzy open) function, then $\left(Y, \tau_{2}\right)$ is a fuzzy mildly normal space.

Theorem 3.6. Let $\left(X, \tau_{1}\right)$ and $\left(Y, \tau_{2}\right)$ be fuzzy topological spaces and let $\left(Y, \tau_{2}\right)$ be a fuzzy mildly normal space (resp. fuzzy normal space). If $f:\left(X, \tau_{1}\right) \rightarrow$ $\left(Y, \tau_{2}\right)$ is a fuzzy almost regular generalized continuous, fuzzy regular closed (resp. fuzzy almost closed) injective function, then $\left(X, \tau_{1}\right)$ is a fuzzy mildly normal space. 
Proof. For each $r \in I_{0}$, let $\lambda_{1}, \lambda_{2} \in I^{X}$ be $r$-rfc sets such that $\lambda_{1} \bar{q} \lambda_{2}$. Since $f$ is a fuzzy regular closed (resp. fuzzy almost closed) injective function, $f\left(\lambda_{1}\right)$ and $f\left(\lambda_{2}\right)$ are $r$-rfc sets (resp. $\tau_{2}\left(f\left(\lambda_{1}\right)^{\prime}\right) \geq r$ and $\left.\tau_{2}\left(f\left(\lambda_{2}\right)^{\prime}\right) \geq r\right)$. Since $\left(Y, \tau_{2}\right)$ is a fuzzy mildly normal (resp. fuzzy normal) space, there exist $\nu_{1}, \nu_{2} \in I^{Y}$ such that $\tau_{2}\left(\nu_{1}\right) \geq r, \tau_{2}\left(\nu_{2}\right) \geq r$ and $f\left(\lambda_{1}\right) \leq \nu_{1}, f\left(\lambda_{2}\right) \leq \nu_{2}$ with $\nu_{1} \bar{q} \nu_{2}$.

Now let $\gamma_{1}=I_{\tau_{2}}\left(C_{\tau_{2}}\left(\nu_{1}, r\right), r\right)$ and let $\gamma_{2}=I_{\tau_{2}}\left(C_{\tau_{2}}\left(\nu_{2}, r\right), r\right)$. Then $\gamma_{1}, \gamma_{2}$ are $r$-rfo sets such that $f\left(\lambda_{1}\right) \leq \gamma_{1}, f\left(\lambda_{2}\right) \leq \gamma_{2}$ and $\gamma_{1} \bar{q} \gamma_{2}$. Since $f$ is a fuzzy almost regular generalized continuous function, then $f^{-1}\left(\gamma_{1}\right)$ and $f^{-1}\left(\gamma_{2}\right)$ are $r$-rgfo sets. Furthermore, $\lambda_{1} \leq f^{-1}\left(\gamma_{1}\right), \lambda_{2} \leq f^{-1}\left(\gamma_{2}\right)$ and $f^{-1}\left(\gamma_{1}\right) \bar{q} f^{-1}\left(\gamma_{2}\right)$. Hence by Theorem $3.1,\left(X, \tau_{1}\right)$ is a fuzzy mildly normal space.

Theorem 3.7. Let $f:\left(X, \tau_{1}\right) \rightarrow\left(Y, \tau_{2}\right)$ be a fuzzy regular continuous, fuzzy almost generalized closed and surjective function. If $\left(X, \tau_{1}\right)$ is a fuzzy mildly normal space, then $\left(Y, \tau_{2}\right)$ is a fuzzy normal space.

Proof. For each $r \in I_{0}$, let $\nu_{1}, \nu_{2} \in I^{Y}$ such that $\tau_{2}\left(\nu_{1}^{\prime}\right) \geq r, \tau_{2}\left(\nu_{2}^{\prime}\right) \geq r$ and $\nu_{1} \bar{q} \nu_{2}$. Since $f$ is fuzzy regular continuous, $f^{-1}\left(\nu_{1}\right)$ and $f^{-1}\left(\nu_{2}\right)$ are $r$-rfc sets with $f^{-1}\left(\nu_{1}\right) \bar{q} f^{-1}\left(\nu_{2}\right)$. Since $\left(X, \tau_{1}\right)$ is fuzzy mildly normal, there exist $\lambda_{1}$, $\lambda_{2} \in I^{X}$ such that $\tau_{1}\left(\lambda_{1}\right) \geq r, \tau_{1}\left(\lambda_{2}\right) \geq r$ and $f^{-1}\left(\nu_{1}\right) \leq \lambda_{1}, f^{-1}\left(\nu_{2}\right) \leq \lambda_{2}$ with $\lambda_{1} \bar{q} \lambda_{2}$.

Let $\mu_{1}=I_{\tau_{1}}\left(C_{\tau_{1}}\left(\lambda_{1}, r\right), r\right)$ and let $\mu_{2}=I_{\tau_{1}}\left(C_{\tau_{1}}\left(\lambda_{2}, r\right), r\right)$. Then clearly $\mu_{1}$ and $\mu_{2}$ are $r$-rfo sets such that $f^{-1}\left(\nu_{1}\right) \leq \mu_{1}, f^{-1}\left(\nu_{2}\right) \leq \mu_{2}$ and $\mu_{1} \bar{q} \mu_{2}$. Since $f$ is fuzzy almost generalized closed, there exist $\gamma_{1}, \gamma_{2} \in I^{Y}$ such that $\gamma_{1}, \gamma_{2}$ are $r$-gfo sets and $\nu_{1} \leq \gamma_{1}, \nu_{2} \leq \gamma_{2}, f^{-1}\left(\gamma_{1}\right) \leq \mu_{1}$ and $f^{-1}\left(\gamma_{2}\right) \leq \mu_{2}$. Since $\mu_{1} \bar{q} \mu_{2}$, then $\gamma_{1} \bar{q} \gamma_{2}$. But $\gamma_{1}, \gamma_{2}$ are $r$-gfo, $\nu_{1} \leq I_{\tau_{2}}\left(\gamma_{1}, r\right)$ and $\nu_{2} \leq I_{\tau_{2}}\left(\gamma_{2}, r\right)$. Furthermore $I_{\tau_{2}}\left(\gamma_{1}, r\right) \bar{q} I_{\tau_{2}}\left(\gamma_{2}, r\right)$. Hence $\left(Y, \tau_{2}\right)$ is a fuzzy normal space.

Corollary 3.8. Let $f:\left(X, \tau_{1}\right) \rightarrow\left(Y, \tau_{2}\right)$ be a fuzzy regular continuous, fuzzy closed and surjective function. If $\left(X, \tau_{1}\right)$ is a fuzzy mildly normal space, then $\left(Y, \tau_{2}\right)$ is a fuzzy normal space.

Theorem 3.9. Let $f:\left(X, \tau_{1}\right) \rightarrow\left(Y, \tau_{2}\right)$ be a fuzzy regular irresolute (resp. fuzzy almost continuous), fuzzy almost regular generalized closed and surjective function. If $\left(X, \tau_{1}\right)$ is a fuzzy mildly normal space (resp. fuzzy normal space), then $\left(Y, \tau_{2}\right)$ is a fuzzy mildly normal space.

Proof. For each $r \in I_{0}$, let $\nu_{1}, \nu_{2} \in I^{Y}$ be $r$-rfc sets such that $\nu_{1} \bar{q} \nu_{2}$. Since $f$ is a fuzzy regular irresolute (resp. fuzzy almost closed ) function, $f^{-1}\left(\nu_{1}\right)$ and $f^{-1}\left(\nu_{2}\right)$ are $r$-rfc sets (resp. $\tau_{1}\left(f^{-1}\left(\nu_{1}\right)^{\prime}\right) \geq r, \tau_{1}\left(f^{-1}\left(\nu_{2}\right)^{\prime}\right) \geq r$ ) such that $f^{-1}\left(\nu_{1}\right) \bar{q} f^{-1}\left(\nu_{2}\right)$. Since $\left(X, \tau_{1}\right)$ is a fuzzy mildly normal space (resp. fuzzy normal space), there exist $\lambda_{1}, \lambda_{2} \in I^{X}$ such that $f^{-1}\left(\nu_{1}\right) \leq \lambda_{1}, f^{-1}\left(\nu_{2}\right) \leq \lambda_{2}$ and $\lambda_{1} \bar{q} \lambda_{2}$.

Let $\mu_{1}=I_{\tau_{1}}\left(C_{\tau_{1}}\left(\lambda_{1}, r\right), r\right)$ and let $\mu_{2}=I_{\tau_{1}}\left(C_{\tau_{1}}\left(\lambda_{2}, r\right), r\right)$. Then clearly $\mu_{1}$, $\mu_{2}$ are $r$-rfo sets such that $f^{-1}\left(\nu_{1}\right) \leq \mu_{1}, f^{-1}\left(\nu_{2}\right) \leq \mu_{2}$ and $\mu_{1} \bar{q} \mu_{2}$. Since $f$ is a fuzzy almost regular generalized closed function, there exist $\gamma_{1}, \gamma_{2} \in I^{Y}$ such that $\gamma_{1}$ and $\gamma_{2}$ are $r$-grfo sets, $\nu_{1} \leq \gamma_{1}, \nu_{2} \leq \gamma_{2}, f^{-1}\left(\gamma_{1}\right) \leq \mu_{1}$ and 
$f^{-1}\left(\gamma_{2}\right) \leq \mu_{2}$. Since $\mu_{1} \bar{q} \mu_{2}$, then $\gamma_{1} \bar{q} \gamma_{2}$. Hence $\left(Y, \tau_{2}\right)$ is a fuzzy mildly normal space.

Corollary 3.10. Let $f:\left(X, \tau_{1}\right) \rightarrow\left(Y, \tau_{2}\right)$ be a fuzzy almost continuous, fuzzy almost closed and surjective function. If $\left(X, \tau_{1}\right)$ is a fuzzy normal space, then $\left(Y, \tau_{2}\right)$ is a fuzzy mildly normal space.

Proof. The proof is determined straightforward.

\title{
References
}

[1] C. L. Chang, Fuzzy topological spaces, J. Math. Anal. Appl. 24 (1968), 182-190.

[2] K. C. Chattopadhyay and S. K. Samanta, Fuzzy topology: fuzzy closure operator, fuzzy compactness and fuzzy connectedness, Fuzzy Sets and Systems 54 (1993), no. 2, 207-212.

[3] Y. C. Kim, A. A. Ramadan, and S. E. Abbas, r-fuzzy strongly preopen sets in fuzzy topological spaces, Mat. Vesnik 55 (2003), no. 1-2, 1-13.

[4] B. Krsteska and Y. C. Kim, Some types of fuzzy normal spaces in Chang's sense, Adv. Fuzzy Sets Syst. 3 (2008), no. 1, 115-129.

[5] T. Kubiak, On fuzzy topologies, Ph. D Thesis, A. Mickiewicz, poznan, 1985.

[6] A. A. Ramadan, S. E. Abbas, and Y. C. Kim, Fuzzy irresolute mappings in smooth fuzzy topological spaces, J. Fuzzy Math. 9 (2001), no. 4, 865-877.

7] A. P.Šostak, On a fuzzy topological structure, Rend. Circ. Mat. Palermo (2) Suppl. No. 11 (1985), 89-103.

[8] L. A. Zadeh, Fuzzy sets, Information and Control 8 (1965), 338-353.

\author{
Department of Mathematics \\ FACULTy OF SCIENCE \\ Assuit UNIVERsity \\ Assuit, EgYpt \\ E-mail address: mazab57@yahoo.com
}

